PAPER Special Section on Technology Trials and Proof-of-Concept Activities for 5G and Beyond

\title{
Performance Evaluation of Downlink Multi-User Massive MIMO with Configurable Active Antenna System and Inter Access Point Coordination in Low-SHF-Band
}

\author{
Yi JIANG $^{\dagger \text { a) }}$, Kenichiro YAMAZAKI ${ }^{\dagger}$, Nonmembers, Toshihiro HAYATA ${ }^{\dagger}$, Member, Kohei IZUI $^{\dagger}$,

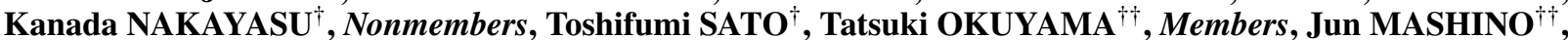 \\ Satoshi SUYAMA ${ }^{\dagger \dagger}$, and Yukihiko OKUMURA ${ }^{\dagger \dagger}$, Senior Members
}

\begin{abstract}
SUMMARY Massive multiple input and multiple output (Massive MIMO) is a key technique to achieve high system capacity and user data rate for the fifth generation $(5 \mathrm{G})$ radio access network (RAN). To implement Massive MIMO in 5G, how much Massive MIMO meets our expectation with various user equipment (UEs) in different environments should be carefully addressed. We focused on using Massive MIMO in the low super-high-frequency (SHF) band, which is expected to be used for $5 \mathrm{G}$ commercial bands relatively soon. We previously developed a prototype low-SHF-band centralized-RAN Massive MIMO system that has a flexible active antenna system (AAS)-unit configuration and facilitates advanced radio coordination features, such as coordinated beamforming (CB) coordinated multi-point (CoMP). In this study, we conduct field trials to evaluate downlink (DL) multi-user (MU)-MIMO performance by using our prototype system in outdoor and indoor environments. The results indicate that about $96 \%$ of the maximum total DL system throughput can be achieved with 1 AAS unit outdoors and 2 AAS units indoors. We also investigate channel capacity based on the real propagation channel estimation data measured by the prototype system. Compared with without-CB mode, the channel capacity of with-CB mode increases by a maximum of $80 \%$ and $104 \%$, respectively, when the location of UEs are randomly selected in the outdoor and indoor environments. Furthermore, the results from the field trial of with-CB mode with eight UEs indicate that the total DL system throughput and user data rate can be significantly improved.

key words: 5G, Massive MIMO, low-SHF-band, MU-MIMO, beamforming, $C-R A N, C B, C o M P$
\end{abstract}

\section{Introduction}

Fifth generation $(5 \mathrm{G})$ commercial services are expected to launch around 2020. Typical scenarios are classified as enhanced Mobile Broad Band (eMBB), Ultra-Reliable and Low Latency Communications (URLLC), and massive Machine Type Communications/massive Internet of Things (mMTC/mIoT). Compared to Long Term Evolution (LTE), these scenarios require, for example, 1000 times the system capacity, delivery of 10-100 times the user experienced data rate, reduction in system latency to $1 \mathrm{~ms}$, and serving 10-100 times the number of terminals with low power consumption [1].

Manuscript received September 7, 2018.

Manuscript revised December 26, 2018.

Manuscript publicized February 20, 2019.

${ }^{\dagger}$ The authors are with the NEC Corporation, Kawasaki-shi, 211-8666 Japan.

${ }^{\dagger}$ The authors are with the NTT DOCOMO, INC, Yokosuka-shi, 239-0847 Japan.

a)E-mail: y-jiang @ct.jp.nec.com

DOI: 10.1587/transcom.2018TTP0013
Among these requirements, a high system capacity and ever-increasing user data rate are two of the most well-known challenges. Massive multiple input and multiple output (Massive MIMO) has attracted significant attention as one of the most promising approaches to increase system capacity and meet user data rate demands of 5G. It uses a very large number of antenna elements at access points (APs) or base stations and simultaneously serves several users with the same time and frequency resources.

Dense small-cell deployment is considered a key strategy for the $5 \mathrm{G}$ network. In such deployment, additional APs are deployed in hot spots where high system capacity and improved user data rate are required. A combination of small cells and Massive MIMO would further meet the requirements in the hot spots. Moreover, multiple neighboring hot spots form a hot zone, expanding coverage. However, such deployment would lead to a severely interference-limited system, requiring careful attention to interference management. If the transmission of neighboring APs can be coordinated, the inter-cell interference among APs can be mitigated.

Although there are many publications on the field trials in the low super-high-frequency (SHF) band, which is the potential candidate frequency band for early commercial services of 5G, [2], [3] focused on the high rank MIMO of a single user equipment (UE), and [4] addressed the throughput performance with fixed TRXs at BS. Some studies, such as [5] and [6], have been made on the performance of coherent joint transmission (JT). In the coherent JT, the antennas used in the beamforming are not colocated but correspond to the locations of APs, and data is transmitted from these noncolocated antennas. [7] mainly discussed a precoding matrix generation algorithm that eliminated the need of channel state information (CSI) exchange between the base stations. However, in with coordinated beamforming (CB) mode of our system, each AP received the SRS not only from its own serving UEs but also the from the CB target UEs which were serving by other AP, therefore, the CSI exchange was not necessary. We have developed a prototype low-SHF centralized radio access network (C-RAN) Massive MIMO system [9][16]. This system has a flexible active antenna system (AAS) configuration in which the number of antennas is configured based on use cases and requirements, such as required cell capacity, communication quality, and UE density per cell. 


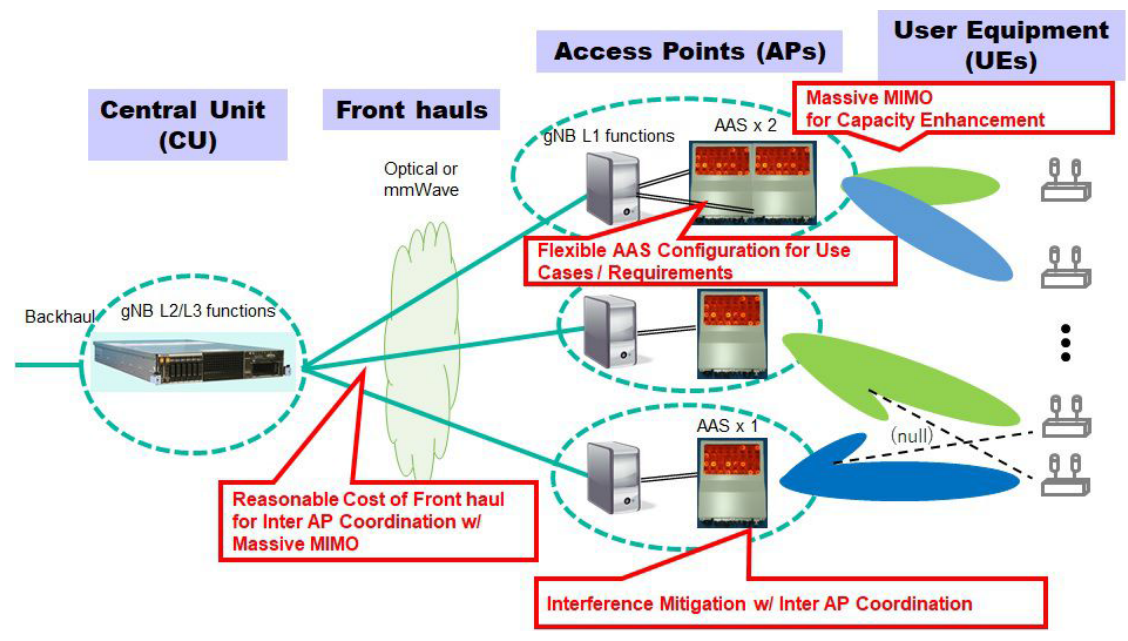

Fig. 1 Block diagram of low-SHF-band C-RAN massive MIMO system.

The C-RAN composition can facilitate advanced radio coordination features, such as coordinated beamforming (CB), Dynamic point selection (DPS), JT, and joint reception (JR) coordinated multi-point (CoMP). In our previous studies, we presented the performance improvement of CB CoMP with up to four UEs in an outdoor environment and evaluated downlink (DL) multi-user (MU)-MIMO performance by using two AAS units in two different outdoor and indoor scenarios [17], [18].

To address the performance of the different scenarios we first evaluated the DL MU-MIMO performance by using our low-SHF-band C-RAN Massive MIMO system with 1 AAS unit and 2 AAS units in outdoor and indoor environments. We then studied the system capacity through simulation based on a real propagation channel. The channel estimation data were measured with 2 AAS units per AP. To confirm throughput enhancement, the field trial on the inter-AP CB with up to eight UEs was conducted.

The rest of this paper is organized as follows. In Sect. 2, we give an overview of our low-SHF band C-RAN Massive MIMO system and the experiment configuration. In Sect. 3, we present the evaluation of the DL MU-MIMO by using our system with 1 AAS and 2 AAS units in outdoor and indoor environments. In Sect. 4, we present the system capacity of CB CoMP using the real propagation channel and present the results of this field trial. The conclusion is given in Sect. 5 .

\section{Low-SHF-Band C-RAN Massive MIMO System}

Figure 1 illustrates a block diagram of our prototype developed low-SHF-band C-RAN Massive MIMO system, and Table 1 lists its specifications. This system consists of a central unit (CU), APs, and UEs. An AP consists of one or more AAS units and digital part (DP) that are connected by multicore multimode optical fibers. The AAS has 64 antenna elements. By connecting two vertical elements to one TRX, 32 transceivers (TRXs) per 1 AAS unit are used. In addition, multiple AAS units can be concatenated vertically and/or horizontally, increasing the number of antenna
Table 1 Specifications of prototype low-SHF-band C-RAN Massive MIMO system.

\begin{tabular}{|c|c|c|}
\hline \multirow{10}{*}{ Common } & Center frequency & $4.55 / 4.65 / 4.75 \mathrm{GHz}$ \\
\hline & Bandwidths & $100 \mathrm{MHz}$ \\
\hline & Duplex & TDD, DL:UL = 9:1 \\
\hline & Multiple access & OFDM \\
\hline & Num. of Subcarriers per PRB & 12 \\
\hline & Num. of PRBs & 125 \\
\hline & Subcarrier spacing & $60 \mathrm{kHz}$ \\
\hline & $\begin{array}{l}\text { Transmission Time Interval (TTI) } \\
\text { period }\end{array}$ & $0.25 \mathrm{msec}$ \\
\hline & Modulation scheme & QPSK, 16QAM, 64QAM \\
\hline & Coding rate & Max. 0.93 \\
\hline $\mathrm{CU}$ & DL link control function & H-ARQ, AMC (w/ OLLA) \\
\hline \multirow[t]{4}{*}{ AP-AAS } & Num. of antenna elements & $\begin{array}{l}1 \text { AAS: } 4 \text { (V) x } 8(\mathrm{H}) \times 2 \\
\text { (Pol.), } 2 \text { AASs: } 4 \text { (V) x } 16 \\
\text { (H) x } 2 \text { (Pol.) }\end{array}$ \\
\hline & Element spacing & $0.52 \lambda(\mathrm{H}), 0.61 \lambda(\mathrm{V})$ \\
\hline & Max. Tx power & $+25 \mathrm{dBm} /$ polarization \\
\hline & Antenna polarization & \pm 45 degrees \\
\hline \multirow{2}{*}{ AP-DP } & DL beamforming algorithm & ZF \\
\hline & Inter AP coordination algorithm & $\mathrm{CB}$ \\
\hline \multirow[t]{3}{*}{ UE } & Antenna configuration & $\begin{array}{l}2 \text { Omni antennas w/ } 3 \lambda \\
\text { Spacing }\end{array}$ \\
\hline & Max. Tx power & $+24 \mathrm{dBm} /$ antenna \\
\hline & Num. of receiver (Rx) layer & Max. 2 \\
\hline
\end{tabular}

elements. The increased number of antenna elements makes the angle resolution of the beam peak and beam null higher. The calibration of the radio frequency chains of the transmitter and receiver at AAS is described in [11] and Inter-AP calibration is not required in the case of CB CoMP. The DP processes the digital signal processing of beamforming and Layer 1 functions. Spatial multiplexing of up to eight UEs, and 16 layers are supported in the field trial. The Uplink (UL) sounding reference signal (SRS) is used by the DP for channel estimation. The channel estimation obtained from the SRS is used for beamforming (BF) weight generation exploiting UL and DL channel reciprocity. Zero-forcing (ZF) precoding is selected as a DL beamforming algorithm [12] and the DL beamforming weight is calculated per physical resource block (PRB). Suppose one AP transmits data sig- 
nal to $K$ UEs. The number of transmit antennas is $N_{T}$ and each UE is equipped with $N_{R}$ antennas. Let $H_{k}$ denote the channel estimation matrix of the $k$ th UE, where $1 \leq k \leq K$. The channel covariance matrix is $\mathbf{H}_{k}^{H} \mathbf{H}_{k}$, where ${ }^{H}$ is Hermitian transpose and its eigenvector is given by $\mathbf{v}_{k, l}$, where $1 \leq l \leq N_{r}$. The $N_{T} * L$ matrix $\mathrm{V}$ is denoted by

$$
\mathbf{V}=\left[\mathbf{v}_{1,1}, \mathbf{v}_{1,2}, \cdots \mathbf{v}_{1, l}, \cdots, \mathbf{v}_{k, 1}, \mathbf{v}_{k, 2}, \cdots \mathbf{v}_{k, l}, \cdots\right]
$$

and with ZF precoding, we get

$$
\mathbf{W}_{\mathbf{Z F}}=\left[\mathbf{w}_{1} \mathbf{w}_{2} \ldots \mathbf{w}_{L}\right]=\mathbf{V}\left(\mathbf{V}^{\mathbf{H}} \mathbf{V}\right)^{-1}
$$

where $L=K \cdot N_{r}$ is the number of Tx layers. The precoding weight is normalized and given by

$$
\mathbf{W}=\left[\mathbf{w}_{1} /\left\|\mathbf{w}_{1}\right\| \mathbf{w}_{2} /\left\|\mathbf{w}_{2}\right\| \cdots \mathbf{w}_{L} /\left\|\mathbf{w}_{L}\right\|\right]
$$

The CU processes Layer 2 and Layer 3 functions. As the interface between the CU and AP is MAC-PHY split and Layer 1 functions are moved from $\mathrm{CU}$ to $\mathrm{AP}$, the required front haul bandwidth between $\mathrm{CU}$ and $\mathrm{AP}$ is reduced to allow 10G Ethernet interface [8], [9]. The CU controls multiple APs located on geographically different points called "sites". The CU schedules the radio resource space domain and controls the hybrid automatic repeat request (H-ARQ) and adaptive modulation and coding (AMC). In AMC, the $\mathrm{CU}$ configures the modulation and coding scheme (MCS) that would result in a block error ratio (BLER) of $10 \%$ on the basis of the outer loop link adaptation (OLLA) in DL.

When inter AP coordination is enabled, APs receives SRS from all UEs involved in the coordination. In the case of DPS, APs calculate the signal-to-interference-plus-noise ratio (SINR) of the received SRS, and sends to CU. The AP with higher received SRS power is selected as the severing AP for the corresponding UE by CU. In the case of $\mathrm{CB}$, the CU sends the MU-MIMO indication message to the APs. This message indicates the target UEs with and without data transmission. The beamforming weight is calculated for all target UEs. Suppose that the number of target UEs without data transmission is $K^{\prime}, L$ in Eq. (2) becomes $\left(K+K^{\prime}\right) * N_{r}$.

A UE consists of TRXs and DP. The antenna spacing is configurable, and in the field trial it is set as 3 wavelengths, as shown in Table 1. Physical uplink shared channel (PUSCH), physical uplink control channel (PUCCH) and SRS are transmitted by UE. In the DL, UE processes signal reception, demodulation, layer separation (up to two layers per UE) and decoding functions. DL channel estimation is calculated per each PRB.

\section{Performance Evaluation of MU-MIMO with 1 AAS Unit and 2 AAS Units}

We used 64 antenna elements (1 AAS unit) or 128 antenna elements (2 AAS units) on the AP-AAS, as shown in Table 1. Note that the transmission power per layer was fixed regardless of the number of multiplexed UEs, and the number of transmission layers per UE was fixed as two. The evaluation was conducted in two different scenarios. One is an outdoor

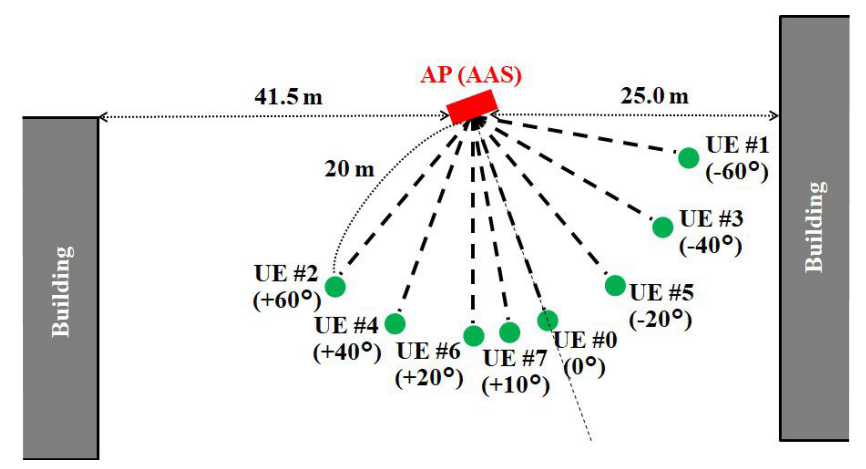

Fig. 2 AP and UE locations in outdoor environment.

parking lot, and the other is an indoor office building. The outdoor environment can be perceived as a large-space outdoor scenario with few reflectors and the indoor environment as a small-space indoor scenario with many reflectors.

\subsection{Outdoor Environment}

The AP (AAS) and UE locations in the outdoor environment are shown in Fig. 2. The UEs were set in a fan-like shape about 20m from the AP. On the basis of the estimated UL channel, the AP calculated the DL BF weights and carried out DL MU-MIMO transmission by changing the number of multiplexed UEs. The time fluctuation in a channel was ignored because UEs were assumed to be stationary. Only one active UE was allocated to location UE \#0, and for the other locations, i.e., UE \#1-7, the AP calculated the DL $\mathrm{BF}$ weights by using the estimated UL channel measured respectively beforehand.

Figure 3 shows the averaged received SINR and DL throughput of UE \#0 in DL MU-MIMO transmission by adding UE \#1 (the farthest from UE \#0) to UE \#7 (the nearest to UE \#0) in ascending order. The $\mathrm{X}$ axis is the number of multiplexed UEs and the left and right $\mathrm{Y}$ axes indicate the averaged received SINR and DL throughput of UE \#0, respectively. For both 1 AAS unit and 2 AAS units, the DL throughput of UE \#0 was around $705 \mathrm{Mbps}$, which is the maximum throughput per UE in this system design, even when the number of multiplexed UEs was eight. Note that the DL throughput of UE reached maximum when the modulation scheme is 64QAM and the coding rate is 0.93 . Although the averaged received SINR of UE\#0 was degraded, it was still high enough to configure the highest modulation order and coding rate. Regarding the received SINR of UE \#0, when the number of multiplexed UEs was one, the SINRs of the 1 AAS unit case and the 2 AAS units case are $29.7 \mathrm{~dB}$ and $30.9 \mathrm{~dB}$, respectively. When the number of multiplexed UEs was eight, the SINRs of the 1 AAS unit case and the 2 AAS units case were 23.2 and $26.5 \mathrm{~dB}$, respectively. The maximum difference between the two cases was $3.3 \mathrm{~dB}$ when the number of multiplexed UEs was eight. The reason is that since the number of antenna element of 2 AAS is twice more than 1 AAS unit, $3 \mathrm{~dB}$ beamforming gain is predictable. Besides, with the double number of antennas, higher degrees of 


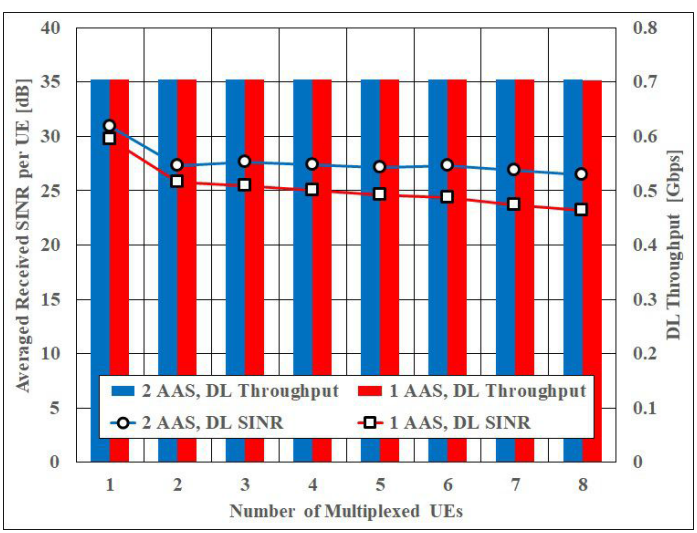

Fig. 3 Averaged received SINR and DL throughput of UE \#0 for 1-8 multiplexed UEs in DL MU-MIMO transmission in outdoor environment.

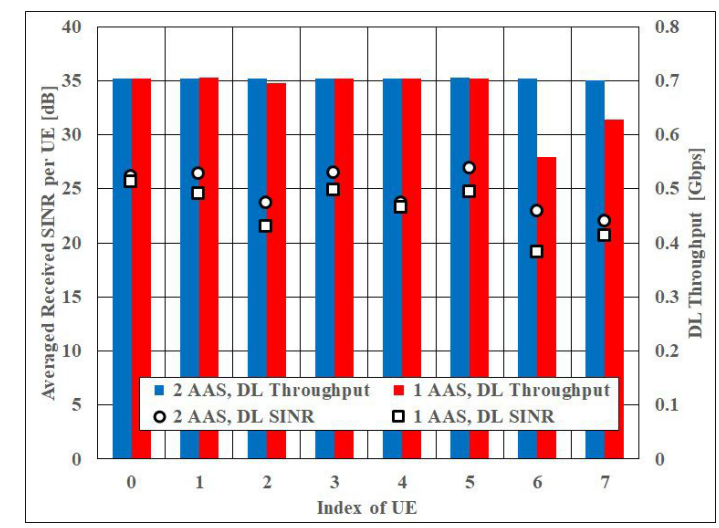

Fig. 4 Averaged received SINR and DL throughput of each of eight multiplexed UEs in DL MU-MIMO transmission in outdoor environment.

freedom can be used for beamforming in the DL MU-MIMO transmission.

We also measured each UE's DL throughput in eight multiplexed UEs case with the same settings. Figure 4 shows the averaged received SINR and DL throughput of each UE in the eight multiplexed UEs DL MU-MIMO case for both the 2 AAS units and the 1 AAS unit. The $\mathrm{X}$ axis is UE index, and the left and right $\mathrm{Y}$ axes indicate the averaged received SINR and the DL throughput of each UE, respectively. The total DL system throughput (i.e. sum of eight UEs) of the 1 AAS unit and the AAS units were 5.4 and $5.6 \mathrm{Gbps}$, respectively. All UEs reached the maximum throughput per UE (705 Mbps) in this system design for 2 AAS units, while the DL throughput of UE \#6 and \#7 degraded for 1 AAS unit. We can confirmed that 2 AAS units mitigated the remaining inter-user-interference because of its narrower beamwidth than that of 1 AAS unit, even when the UEs were closer such as UE \#6 and \#7. In the case of 1 AAS unit, although UE \#0 and \#7 are as close as UE \#6 and \#7, the channel correlation between UE \#0 and \#7 $(-16.8 \mathrm{~dB})$ was about $7 \mathrm{~dB}$ lower than that between UE \#6 and \#7 ( $-9.6 \mathrm{~dB})$. Such relatively high channel correlation caused more remaining inter-user interference during the precoding. This is one of reasons why the performance of UE \#0 is better than that of UE \#6

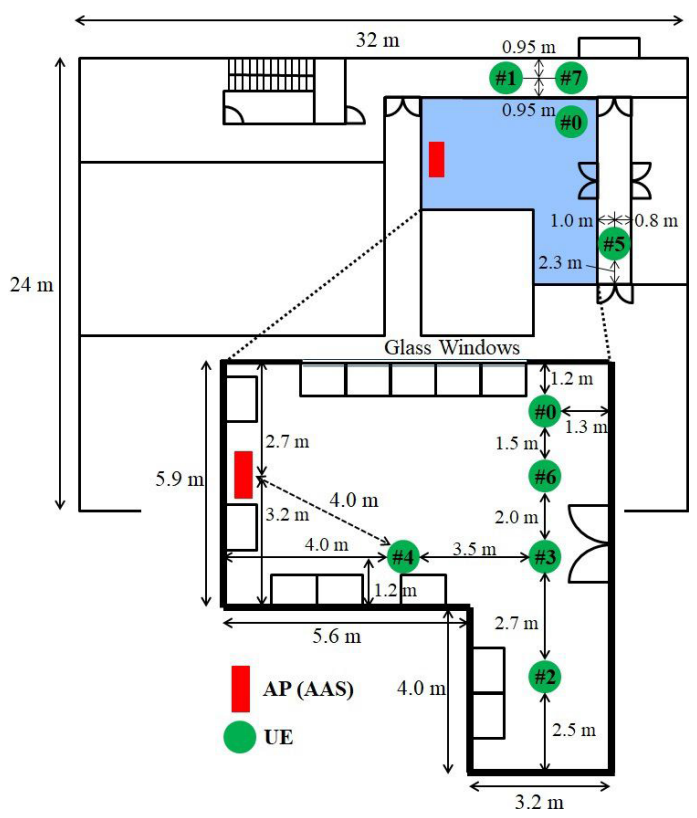

Fig.5 AP (AAS) and UE locations in indoor environment.

and \#7.

\subsection{Indoor Environment}

The AP (AAS) and UE locations in the indoor environment are illustrated in Fig. 5. UE \#0, \#2, \#3, \#4, and \#6 were located in the same room with the AP. UE \#0,\#3, \#4, and \#6 were located in the perspective of AP (LOS), and UE \#2 was located outside the perspective of AP (NLOS). The other UEs (UE \#1, \#5, and \#7) were located outside the room with the AP.

Figure 6 shows the average received SINR and DL throughput of UE \#0 in DL MU-MIMO transmission by adding UE \#1 to \#7 in ascending order. The $X$ axis is the number of multiplexed UEs, and the left and right $Y$ axes indicate the averaged received SINR and the DL throughput of UE \#0, respectively. For 2 AAS units, the DL throughput of UE \#0 remained at about $705 \mathrm{Mbps}$ even when the number of multiplexed UEs increased up to eight. The average received SINR of UE \#0 varied from 30.9 to $24.6 \mathrm{~dB}$ as the number of multiplexed UEs increased. Although the averaged received SINR of UE \#0 degraded, it was still high enough to configure the highest modulation order and coding rate. For 1 AAS unit, however, the DL throughput of UE \#0 decreased to $564 \mathrm{Mbps}$, and the average received SINR changed from 29.7 to $20.4 \mathrm{~dB}$. The difference in the average received SINR tended to become large with the increase in the number of multiplexed UEs, and the maximum difference between the two cases is $4.2 \mathrm{~dB}$ when the number of multiplexed UEs was eight.

Figure 7 shows the averaged received SINR and DL throughput of each UE of the eight multiplexed UEs in DL MU-MIMO transmission. The $\mathrm{X}$ axis is UE index, and the left and right $\mathrm{Y}$ axes are the averaged received SINR 


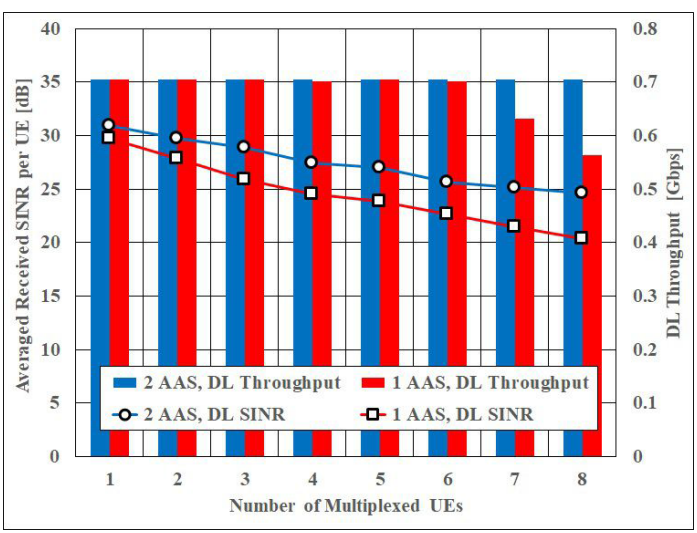

Fig. 6 Averaged received SINR and DL throughput of UE \#0 for 1-8 multiplexed UEs in DL MU-MIMO transmission in indoor environment.

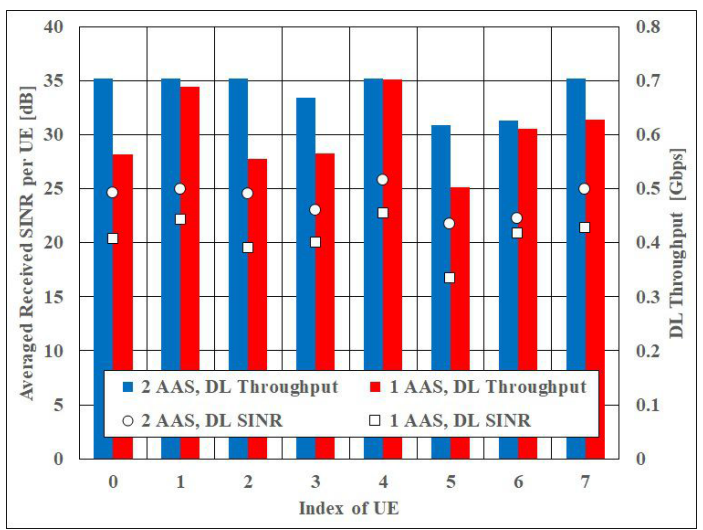

Fig. 7 Averaged received SINR and DL throughput of each UE in eight multiplexed UEs DL MU-MIMO transmission in indoor environment.

and the DL throughput of each UE, respectively. The total DL system throughput (i.e. sum of eight UEs) was $5.4 \mathrm{Gbps}$ (about $96 \%$ of the maximum total DL system throughput in this system design) for 2 AAS units and $4.8 \mathrm{Gbps}$ for $1 \mathrm{AAS}$ unit. We could confirm a greater performance improvement with 2 AAS units compared with that of 1 AAS unit. For 2AAS units, the DL throughput of UE \#2 was higher than that of UE \#3. Since the distances between the AAS and the UEs were relatively small, the transmission power was strong enough to reach the maximum DL throughput if the number of multiplex UE was 1 and the free space propagation was not the main impact on the performance. For instance, although UE \#3 was closer to the AAS than UE \#2, the DL throughput of UE \#3 was lower than that of UE \#2. The similar phenomena was observed at UE \#1 compared to the UE indoors, such as UE \#0, \#2, \#3 and \#6.

We now consider the DL MU-MIMO evaluation results of both the outdoor environment and indoor environment. Table 2 shows the delay spread of each UE and averaged delay spread over all UEs of the outdoor environment and indoor environments. We also analyzed the averaged channel correlation of each UE, as shown in Table 3. For an instance, for UE\#0, it is the average of the channel correla-
Table 2 Delay spread of outdoor and indoor environment [ns].

\begin{tabular}{|l|l|l|l|l|l|l|l|l|l|}
\hline UE & $\# 0$ & $\# 1$ & $\# 2$ & $\# 3$ & $\# 4$ & $\# 5$ & $\# 6$ & $\# 7$ & Ave. \\
\hline outdoor & 35.3 & 34.7 & 58.5 & 33.9 & 38.7 & 37.2 & 44.4 & 51.7 & 41.8 \\
\hline indoor & 41.8 & 45.5 & 49.0 & 50.6 & 51.1 & 72.0 & 50.0 & 45.6 & 50.7 \\
\hline
\end{tabular}

Table 3 Averaged channel correlation between UEs [dB].

\begin{tabular}{|l|l|l|l|l|l|l|l|l|l|}
\hline UE & $\# 0$ & $\# 1$ & $\# 2$ & $\# 3$ & $\# 4$ & $\# 5$ & $\# 6$ & $\# 7$ & Ave. \\
\hline \multicolumn{10}{|c|}{ outdoor } \\
\hline 1 AAS & -18 & -18 & -20 & -17 & -19 & -20 & -16 & -16 & -18 \\
\hline 2 AAS & -22 & -20 & -20 & -19 & -21 & -25 & -20 & -19 & -20 \\
\hline \multicolumn{10}{|c|}{ indoor } \\
\hline 1 AAS & -17 & -15 & -14 & -15 & -16 & -15 & -18 & -16 & -16 \\
\hline 2 AAS & -20 & 18 & 17 & -17 & -21 & -18 & -20 & -19 & -19 \\
\hline
\end{tabular}

tions between UE\#0 and any other UE, \#1-\#7. In the indoor environment, delay spread was larger than in the outdoor environment, and channel correlation was higher by about $2 \mathrm{~dB}$ than the outdoor environment. Due to the larger area in the outdoor environment, the locations of UE can be selected with lower channel correlation, even in 1 AAS unit, about $96 \%$ of the maximum total DL system throughput was reached, whereas about $86 \%$ of the maximum total DL system throughput was reached in the indoor environment. We confirmed that our prototype low-SHF-band Massive MIMO system can optimize the DL throughput performance with the minimum configuration of AAS units by changing the configuration appropriately depending on the environment.

\section{Performance Evaluation of Inter AP Coordination}

\subsection{Simulation Results of Channel Capacity with Real Propagation Channel Estimation Data}

In this section, the number of AAS units per AP during channel measurement in both outdoor and indoor environments are two. Two APs and one UE were used. The coverage of two APs can be perceived as a two-site deployment scenario. During the measurement, the SRS was received by both AP\#1 and \#2 to allow for APs to estimate the propagation channel. The channel-estimation data were recorded at both APs and used as the input for the simulation. The locations of UEs were chosen randomly in the simulation, i.e., no scheduling algorithm for UE combinations was included. Before BF weight calculation, additive white Gaussian noise (AWGN) of a signal-to-noise ratio $(\mathrm{SNR})=20 \mathrm{~dB}$ was added to the measured channel data to simulate the channel-estimation error using UL/DL reciprocity and AAS calibration error. In our prototype system, the maximum DL throughput per UE can be achieved when the SNR is higher than $20 \mathrm{~dB}$. The channel capacity was calculated using Shannon's theorem.

The following four modes were simulated. In the multiAP mode (mode 1 and 2 below), one AP is selected as the serving AP, in which the received power SRS is higher. Only the serving AP transmits DL data to the UE. 1) CB mode (w/ CB): both APs calculate the ZF BF weights for all UEs. Therefore, the DL interference from the non-serving 


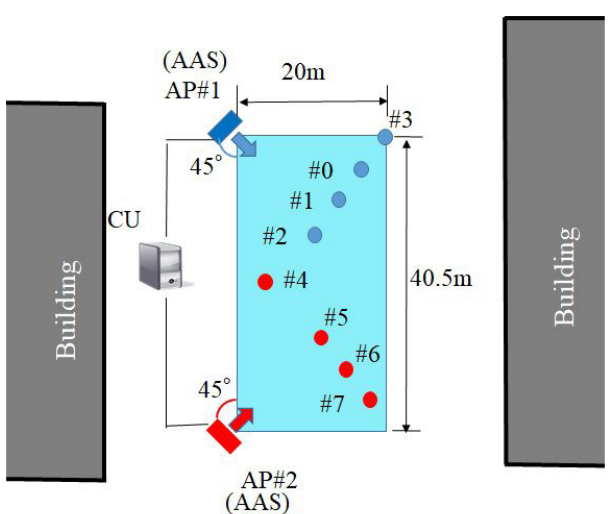

Fig. 8 APs and UEs locations in simulations and the filed trial of CB.

$\mathrm{AP}$ is reduced. However, more freedom of $\mathrm{BF}$ weights is consumed than that in non-CB mode. 2) non-CB mode (w/o $\mathrm{CB}$ ): each AP calculates the ZF BF weights for serving UEs only. Therefore, the interference from the non-serving AP is not reduced. 3) AP\#1 only mode: all UEs are served by AP\#1 and AP\#2 is not used. 4) AP\#2 only mode: All UEs are served by AP\#2 and AP\#1 is not used.

\subsubsection{Outdoor Environment}

The height of the AAS and the antennas at the UE were 3 and $1.7 \mathrm{~m}$, respectively. Figure 8 illustrates an overview of the measurement area and orientations of the AAS. The UE was located with LOS to the AAS. About 9*1200 points in the blue area are measured and the interval between two adjacent points in the direction of $20 \mathrm{~m}$ is about $2 \mathrm{~m}$ while about $0.03 \mathrm{~m}$ in the direction of $40.5 \mathrm{~m}$. The channel capacity (bps/Hz) vs. the number of UEs in AP\#1 only, AP\#2 only, w/o CB and w/ CB is shown in Fig. 9. Compared to AP\#1 only, AP\#2 only and w/o $\mathrm{CB}$, the channel capacity in $\mathrm{w} / \mathrm{CB}$ increases 46\% (AP\#1 only), 39\% (AP\#2 only) and 80\%, respectively. The system capacity reached maximum when the number of UEs was 18. Figure 10 illustrates the cumulative distribution function (CDF) of the received signal-to-interference power ratio (SIR) when the number of UE was 18. The SIR was the highest in w/ CB.

\subsubsection{Indoor Environment}

The height of an AAS and the antennas at the UE were 2.5 and $1.7 \mathrm{~m}$, respectively. Figure 11 illustrates APs and UE locations in the indoor environment for channel-capacity simulation. The orientations of the APs are shown as green dotted lines. About 3275 points in the blue area are measure and the interval between two adjacent points is about $0.02 \mathrm{~m}$. Both LOS and NLOS conditions existed. Figure 12 illustrates channel capacity (bps/Hz) vs. the number of UEs in AP\#1 only, AP\#2 only, w/o CB and w/ CB. Compared to AP\#1 only, AP\#2 only and w/o CB, the channel capacity in w/ CB increases $37 \%$ (AP\#1 only), 26\% (AP\#2 only) and $104 \%$, respectively. The system capacity reached maximum when the number of UEs was 22. Figure 13 illustrates the

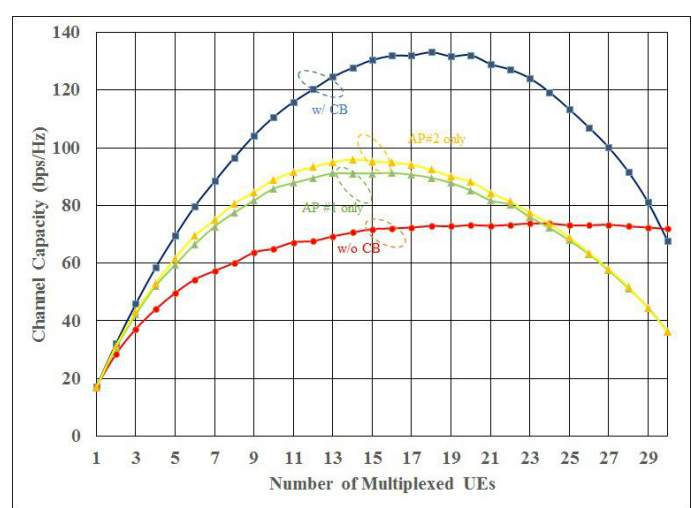

Fig. 9 Estimated channel capacity for AP\#1 only, AP\#2 only, w/o CB and $\mathrm{w} / \mathrm{CB}$ in outdoor environment.

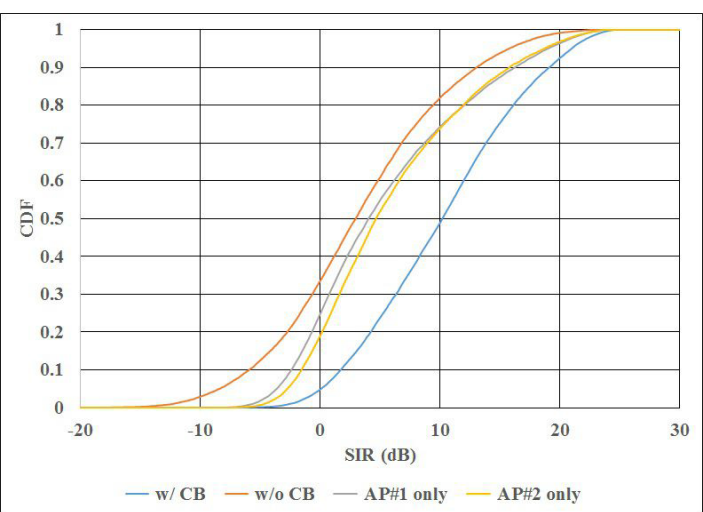

Fig. 10 CDF of received SIR in AP\#1only, AP\#2 only, w/o CB and w/ $\mathrm{CB}$ when number of UEs was 18 in outdoor environment.

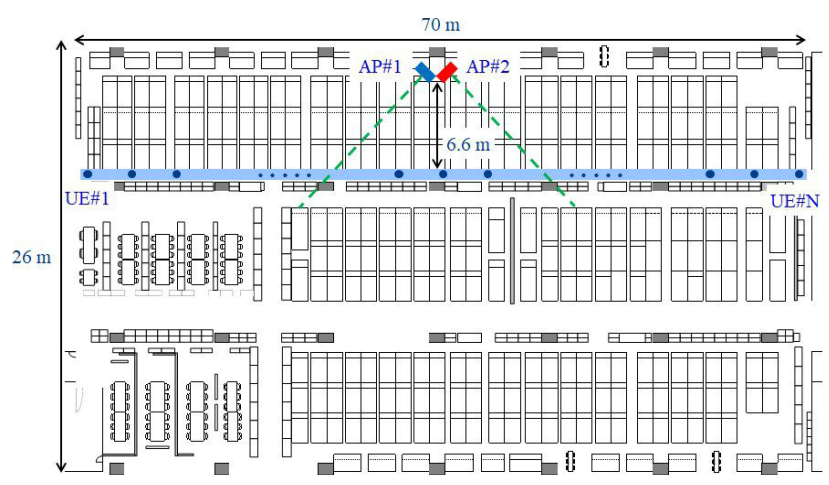

Fig. 11 APs and UE locations in simulation in indoor environment.

CDF of received SIR when the number of UEs was 22.

Additionally, we illustrated the maximum channel capacity for the case of 1 AAS unit per AP in both outdoor and indoor environment and compared it with the case of 2AAS units per AP. The comparison results are shown in Fig. 14. The numbers on the top of each bar indicate the number of multiplexed UEs in each different cases. As 2AAS units employed twice more antenna elements, the number of multiplexed UE that it can support will be doubled. In both outdoor and indoor environments, the maximum estimated channel capacity of 2 AAS units is about 1.8-1.9 times more 


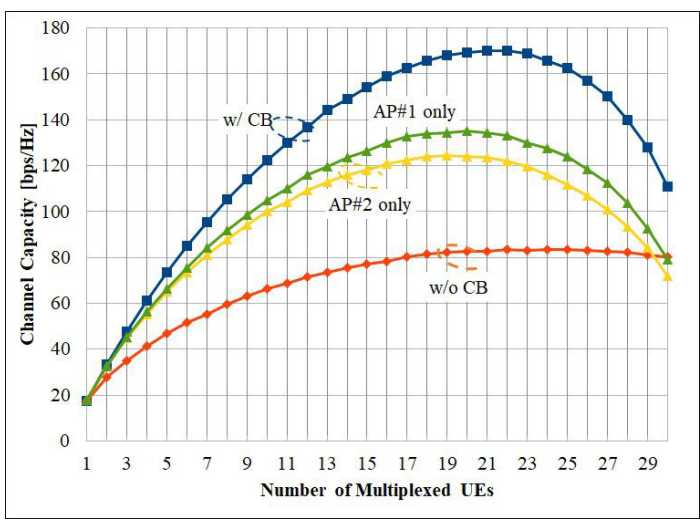

Fig. 12 Estimated channel capacity in AP\#1 only, AP\#2 only, w/o CB and $\mathrm{w} / \mathrm{CB}$ in indoor environment.

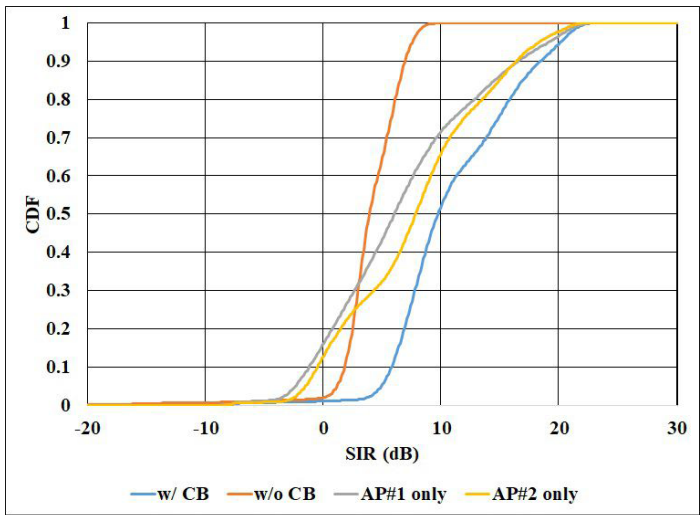

Fig. 13 CDF of received SIR in AP\#1 only, AP\#2 only, w/o CB and w/ $\mathrm{CB}$ when number of UEs is 22 in indoor environment.

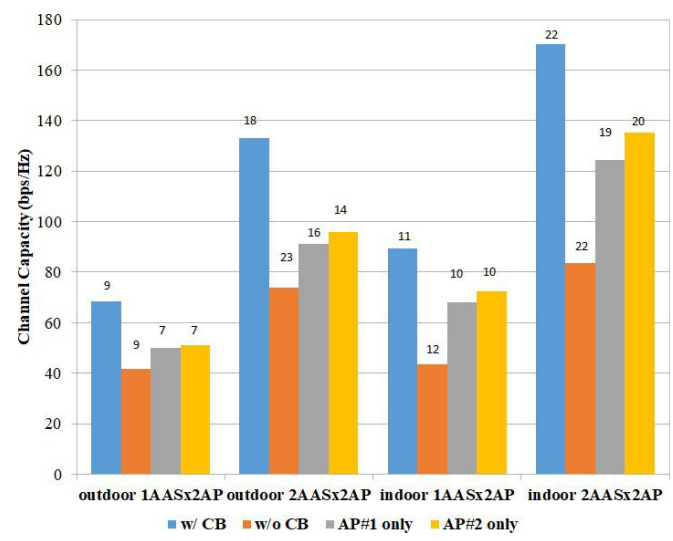

Fig. 14 Comparison of maximum estimated channel capacity with 1 AAS and 2 AAS units.

than that of 1 AAS unit, as we expected. Besides, the maximum estimated channel capacity of $\mathrm{w} / \mathrm{CB}$ in the outdoor environment is about 1.6-1.8 times than that of w/o CB, while it is about 2 times in the indoor environment. The reason is that because of different directions of two AASs, the inter-cell interference area between two APs is wider in the indoor environment than in the outdoor environment, therefore the enhancement from $\mathrm{w} / \mathrm{CB}$ is larger in the in-
Table 4 DL throughput (TP) in AP\#1 only, AP\#2 only, w/o CB and w/ $\mathrm{CB}$ [Mbps].

\begin{tabular}{|l|l|l|l|l|l|l|l|l|l|}
\hline DL TP & $\# 0$ & $\# 1$ & $\# 2$ & $\# 3$ & $\# 4$ & $\# 5$ & $\# 6$ & $\# 7$ & Total \\
\hline w/ CB & 654 & 519 & 702 & 681 & 703 & 705 & 576 & 701 & 5241 \\
\hline w/o CB & 562 & 555 & 594 & 627 & 501 & 559 & 585 & 560 & 4543 \\
\hline AP\#1 Only & 612 & 486 & 664 & 627 & 557 & 243 & 189 & 139 & 3517 \\
\hline AP\#2 Only & 151 & 154 & 129 & 91 & 701 & 686 & 538 & 641 & 3091 \\
\hline
\end{tabular}

Table 5 Averaged received SINR in AP\#1 only, AP\#2 only, w/o CB and $\mathrm{w} / \mathrm{CB}[\mathrm{dB}]$.

\begin{tabular}{|l|l|l|l|l|l|l|l|l|}
\hline $\begin{array}{l}\text { Averaged Received } \\
\text { SINR }\end{array}$ & $\# 0$ & $\# 1$ & $\# 2$ & $\# 3$ & $\# 4$ & $\# 5$ & $\# 6$ & $\# 7$ \\
\hline w/ CB & 22 & 19 & 24 & 22 & 24 & 24 & 21 & 25 \\
\hline w/o CB & 19 & 20 & 20 & 21 & 17 & 19 & 20 & 21 \\
\hline AP\#1 Only & 21 & 17 & 22 & 21 & 19 & 8 & 5 & 4 \\
\hline AP\#2 Only & 4 & 4 & 3 & 0 & 24 & 23 & 18 & 23 \\
\hline
\end{tabular}

door environment. Moreover, in both cases of 1 AAS unit and 2AAS units, the maximum estimated channel capacity of the outdoor is smaller than that of the indoor. The reason is that generally the number of multipath components was large in the indoor environment, so that the channel correlations between UEs are relatively low and the antenna degrees of freedom can be used more efficiently even without the deliberated selection of multiplexed UEs. Besides, we can observe that in the $1 \mathrm{AP}$ only (2AAS/AP) mode, the maximum channel capacity is larger than in w/ CB (1AAS/AP) mode.

\subsection{Field Trial of Inter-AP Coordination in Outdoor Envi- ronment}

Due to the hardware constraints on the number of UEs, the performance evaluation of inter-AP coordination was conducted with up to eight UEs. Two APs and one AAS per AP were used. All APs and UEs were active and stationary during the measurement. The orientations of the AAS are the same as in 4.1.1, as shown in Fig. 8. Similar with the simulations, the following four modes, $w / \mathrm{CB}$, w/o CB, $\mathrm{AP} \# 1$ only and $\mathrm{AP} \# 2$ only were evaluated. In the modes of w/ CB and w/o CB, UE \#0-\#3 were served by AP\#1 while UE \#4-\#7 were served by AP\#2. All UEs were served by AP\#1 and AP\#2 was not used in the AP\#1 only mode, while all UEs were served by AP\#2 and AP\#1 was not used in the AP\#2 only mode.

The DL throughput in w/ CB, w/o CB, AP\#1 only, and AP\#2 only is shown in Table 4. In w/ CB, the total DL throughput was about $5.2 \mathrm{Gbps}$, while the maximum throughput of one UE reached around $705 \mathrm{Mbps}$. Compared with the w/o CB, the total DL throughput increase was about $700 \mathrm{Mbps}$, which is an increase of about $15 \%$. Table 5 shows the average received SINR at each UE. The average received SINR of all UEs except UE\#1 increased in w/ CB, and the maximum enhancement per UE was up to $6.5 \mathrm{~dB}$. We also calculated channel capacity based on the received SINR of each layer of each UE by using Shannon's theorem and the enhancement of channel capacity in w/ CB increased 
by about $15 \%$, which is consistent with that of the total DL throughput. Such enhancement of channel capacity $(15 \%)$ in the field trial is smaller than that of the simulations. The reason is that the UE locations was just one snapshot sample during the field trial while they were several snapshot samples and the channel capacity was averaged in the simulations. For the same reason, the total DL throughput in $1 \mathrm{AP}$ only is lower than in w/o CB in the field trial.

We also observed that the averaged received SINR of UE \#1 slightly degraded in w/ CB compared to that in w/o $\mathrm{CB}$. The reason is that the reduction of $\mathrm{S}$ was larger than that of I+N. Since the spatial channel correlations of UE $\# 1$ and \#6 and UE \#1 and \#7 were relatively high, some of the transmission signal power was used to remove the interference signal from UE \#1 in the ZF precoding. This decreased the transmission power to the direction of UE \#1 (eigen vector direction of UE \#1) in w/ CB. The reason that the enhancement in the average received SINR of UE \#6 was relatively small is similar. Due to the high spatial channel correlation of UE \#6 and \#0, the transmission power toward to UE \#6 also decreased in w/ CB.

\section{Conclusion}

In this paper, we evaluated the performance of DL MUMIMO by using a prototype low-SHF-band C-RAN Massive MIMO System. The field trials were conducted in the outdoor and indoor environment. We confirmed that with 1 AAS unit in the outdoor and 2 AAS units in the indoor environment the total DL system throughput achieves $5.4 \mathrm{Gbps}$ which is about $96 \%$ of the maximum total DL throughput in the system design with the bandwidth up to $100 \mathrm{MHz}$. The prototype system can optimize the performance of DL throughput and user data rate with the minimum configuration of AAS units by changing the configuration appropriately depending on the environment. We also investigated the channel capacity using a real propagation channel through the simulations. In the case of 2AAS units per AP, the channel capacity in CB increases by a maximum of $80 \%$ and $104 \%$ with random UE locations selections, compared to w/o CB in the outdoor and indoor environment, respectively. The maximum estimated channel capacity of 2 AAS units per AP is about 1.8-1.9 times more than that of 1 AAS unit per AP. Moreover,the maximum estimated channel capacity of the outdoor is smaller than that of the indoor. In the indoor environment, the antenna degrees of freedom can be used more efficiently even without the deliberated selection of multiplexed UEs. Furthermore, for the field trial w/ CB, the results show that the DL throughput at each UE can be improved greatly.

\section{Acknowledgments}

This technical paper includes a part of results of "The research and development project for realization of the fifthgeneration mobile communications system" commissioned by The Ministry of Internal Affairs and Communications,
Japan.

\section{References}

[1] "ARIB 2020 and Beyond Ad Hoc Group White Paper," Mobile Communications Systems for 2020 and beyond, v 1.0, Oct. 2014.

[2] Y. Inoue, S. Yoshioka, Y. Kishiyama, T. Haruna, T. Tanaka, A. Splett, S. Suyama, Y. Okumura, and H. Liljestrom, "Field experimental evaluation of low SHF 5G radio access system employing higher rank MIMO,” 2017 IEEE 85th Vehicular Technology Conference (VTC Spring), pp.1-5, Sydney, NSW, June 2017.

[3] Y. Takahashi, Y. Inoue, S. Yoshioka, Y. Kishiyama, S. Suyama, J. Mashino, Y. Okumura, T. Haruna, T. Tanaka, A. Splett, and H. Liljestrom, "Field experimental evaluation of higher rank MIMO in quad-directional UE antenna configuration for $5 \mathrm{G}$ radio access system," 2017 20th International Symposium on Wireless Personal Multimedia Communications (WPMC), pp.542-546, Bali, 2017.

[4] Y. Saito, A. Benjebbour, Y. Kishiyama, X. Wang, X. Hou, H. Jiang, L. Lu, W. Liang, B. Li, L. Gu, Y. Cui, and T. Kashima, "Large scale field experimental trial of downlink TDD Massive MIMO at the 4.5 GHz band," 2017 IEEE 85th Vehicular Technology Conference (VTC Spring), pp.1-5, Sydney, NSW, June 2017.

[5] H. Seki, M. Tsutsui, M. Minowa, K. Shiizaki, C. Akiyama, T. Okuyama, J. Mashino, S. Suyama, and Y. Okumura, "Field experiment of high-capacity technologies for $5 \mathrm{G}$ ultra high-density distributed antenna systems," 2017 IEEE 85th Vehicular Technology Conference (VTC Spring), pp.1-5, Sydney, NSW, June 2017.

[6] T. Okuyama, S. Suyama, J. Mashino, Y. Okumura, K. Shiizaki, C. Akiyama, M. Tsutsui, H. Seki, and M. Minowa, "Antenna deployment of 5G ultra high-density distributed Massive MIMO by low-SHFband indoor and outdoor experiments," 2017 IEEE 86th Vehicular Technology Conference (VTC Fall), pp.1-5, Toronto, Canada, Sept. 2017.

[7] K. Hoshino and M. Mikami, "Experimental evaluation of simple precoding technique for multi-cell coordinated MU-MIMO," 2017 IEEE 85th Vehicular Technology Conference (VTC Spring), pp.1-5, Sydney, NSW, June 2017.

[8] Y. Maruta and T. Sato, "Study on the front-haul for mobile radio access networks with massive antennas," IEICE Society Conference, BT-1-3, Sept. 2014.

[9] Y. Maruta, T. Sato, and Y. Kakura, "Activities on high capacity 5G wireless access," IEICE Technical Report, RCS2015-173, Oct. 2015.

[10] T. Sato, "A view on Massive MIMO and C-RAN toward 5G," IEICE Technical Report, RCS2016-216, Dec. 2016.

[11] T. Mochizuki, et al, "Development of $4.65 \mathrm{GHz}$ band massive element active antenna for 5G," IEICE General Conference, B-1-119, March 2018.

[12] J. Shikida, et al, "Performance evaluation of low complexity multiuser MIMO scheduling schemes for Massive MIMO system," IEICE Technical Report, RCS2015-173, Oct. 2015

[13] T. Okuyama, et al., "Performance evaluation of digital beamforming by indoor and outdoor propagation experimental results for 5G Massive MIMO in low SHF band," IEICE Technical Report, RCS201725, April 2017.

[14] K. Yamazaki, T. Sato, Y. Maruta, T. Okuyama, J. Mashino, S. Suyama, and Y. Okumura, "DL MU-MIMO field trial with 5G low SHF band Massive MIMO antenna," 2017 IEEE 85th Vehicular Technology Conference (VTC Spring), pp.1-5, Sydney, NSW, June 2017.

[15] D. Nose, et al., "Outdoor DL MU-MIMO and inter access point coordination performance of low-SHF-band C-RAN Massive MIMO system for 5G," IEICE Technical Report, RCS2017-353, Feb. 2018.

[16] Y. Maruta, K. Yamazaki, K. Izui, T. Okuyama, J. Mashino, S. Suyama, K. Nakayasu, T. Sato, and Y. Okumura, "Outdoor DL MU-MIMO and inter access point coordination performance of lowSHF-band C-RAN Massive MIMO system for 5G,” 2018 IEEE 87th Vehicular Technology Conference (VTC Spring), pp.1-5, Porto, June 
2018

[17] Jiang, et al., "Indoor and outdoor field experimental results of lowSHF-band C-RAN Massive MIMO system for 5G," RCS2018-24, May 2018

[18] K. Yamazaki, K. Izui, K. Nakayasu, T. Okuyama, J. Mashino, S. Suyama, T. Sato, and Y. Okumura, "Field experimental DL MUMIMO evaluations of low-SHF-band C-RAN Massive MIMO system with over 100 antenna elements for 5G," 2018 IEEE 88th Vehicular Technology Conference (VTC Fall), pp.1-5, Chicago, USA, Aug. 2018

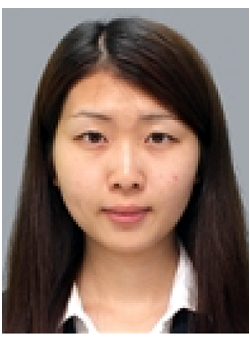

Yi Jiang received the B.S. from Beijing Institute of Petrochemical Technology in 2006, the M.S. degrees from Beijing University of Post and Telecommunication, Beijing, China, in 2010, and the Ph.D. degrees from Waseda University, Tokyo, Japan in 2013. She joined NEC in 2013. Currently, She is involved in research and development of 5G Massive MIMO system. She received the Young Researcher's Award of IEICE in 2011.

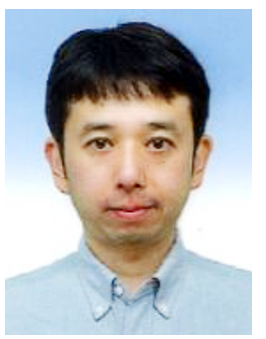

Kenichiro Yamazaki received the B.S. and M.S. degrees from Hokkaido University, Sapporo, Japan, in 1996 and 1998, respectively. He joined Mitsubishi Electric Corporation in 1998. He has been engaged in research and development on digital mobile radio communication systems. Since September 2006, he has joined NEC Corporation and is involved in research and development of wireless access technologies including experimental evaluations for LTE-Advanced system and 5G Massive MIMO system. He received the Young Researcher's Award of IEICE in 2004.

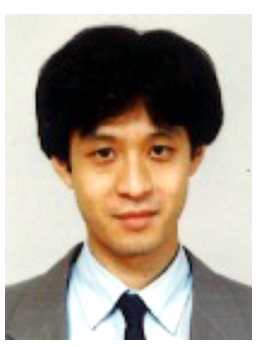

Toshihiro Hayata received the B.S. and M.S. degrees in Electrical Engineering from the University of Tokyo in 1989 and 1991, respectively. He joined NEC in 1991. Since 1993 he is involved in the development of $3 \mathrm{G}$ and $4 \mathrm{G}$ mobile radio base stations. He is a member of IEICE.

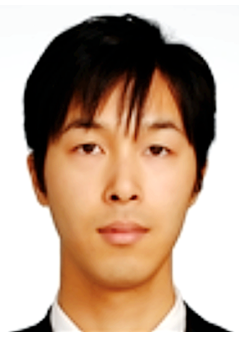

Kohei Izui received the B.S. and M.S. degrees from Hiroshima City University, hiroshima, Japan, in 2014 and 2016, respectively. He joined NEC Corporation in 2016, and is involved in development of wireless access technologies including experimental evaluations for 5G Massive MIMO system.

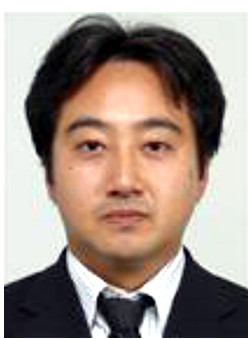

Kanada Nakayasu

received his B.S. degrees from the Nagoya Institute of Technology, Japan, in 1993. He joined NEC in 1993. He has been involved in the development of $2 \mathrm{G}, 3 \mathrm{G}$ and $4 \mathrm{G}$ mobile base station systems, especially the hardware and software design for baseband signal processing and call processing of base satations. He is now the member of the $5 G$ R\&D Massive MIMO project in NEC.

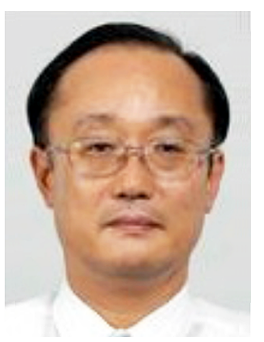

Toshifumi Sato received his B.E. and M.E degrees in electronic engineering from the University of Tokyo, Japan, in 1979 and 1981, respectively. He joined NEC in 1981. Since 1990 he is involved in the development of $2 \mathrm{G}, 3 \mathrm{G}$ and $4 \mathrm{G}$ mobile radio access systems, especially their base station architecture and signal processing units. He is now an active member of the $5 \mathrm{G}$ Massive MIMO R\&D project in NEC as an executive specialist. He is a member of IEEE and IEICE.

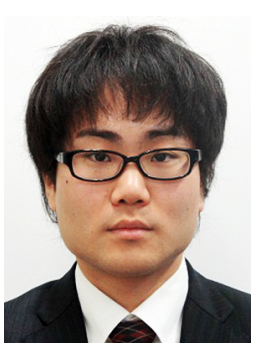

Tatsuki Okuyama received the B.S. degree in electrical, electronic and information engineering and M.S. degree in information and communications technology, all from Osaka University, Osaka, Japan in 2012 and 2014 respectively. Since April 2014, he has been with NTT DOCOMO, INC. He has been engaged in research of fifth generation mobile communications system. He received the Young Researchers' Award from IEICE in 2017. He is a member of IEICE.

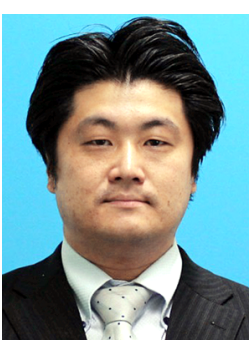

Jun Mashino received the B.E. degree in electrical and electronic engineering and M.E. degree in communications and computer engineering from Kyoto University, Japan in 2003 and 2005, respectively. He joined NTT Access Network Service Systems Laboratories, NTT Corporation in 2005 . He has been engaged in the research and development of intelligent interference compensation technologies, and signal processing for future wireless communication systems. Since 2016, he has been working as a research engineer in NTT DOCOMO 5G Laboratory. His current interests include wireless transmission technologies for $5 \mathrm{G}$ fifth generation mobile communications system. He received the IEICE Young Engineers Award in 2009 and also received APMC2014 Prize in 2014. He is a senior member of IEICE and a member of IEEE. 


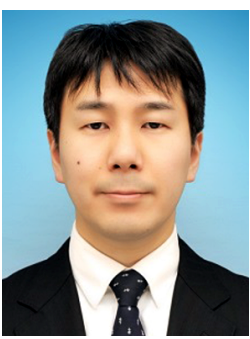

Satoshi Suyama received the B.S. degree in electrical and electronic engineering, the M.S. degree in information processing, and the Dr. Eng. degree in communications and integrated systems, all from Tokyo Institute of Technology, Tokyo, Japan, in 1999, 2001, and 2010, respectively. From 2001 to 2013, he was an Assistant Professor in the Department of Communications and Integrated Systems at the Tokyo Institute of Technology. He has been engaged in research on OFDM mobile communications systems and applications of the adaptive signal processing, including turbo equalization, interference cancellation, and channel estimation. Since April 2013, he has joined NTT DOCOMO, INC. and is involved in research and development of fifth generation mobile communications system. Dr. Suyama is a member of IEEE and a senior member of the Institute of Electronics, Information, and Communication Engineers (IEICE) of Japan. He received the Young Researchers' Award from IEICE in 2005, the Best Paper Prize from the European Wireless Technology Conference (EuWiT) in 2009, Best Paper Award from International Symposium on Personal, Indoor and Mobile Radio Communications (PIMRC) in 2016, and the Paper Award from IEICE in 2012.

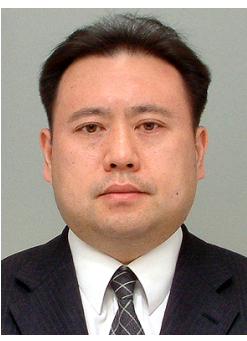

Yukihiko Okumura received his M.S. degree in electrical engineering from the Tokyo University of Science, Tokyo, Japan, in 1991, and his Ph.D. degree in engineering from the Tohoku University, Miyagi, Japan, in 2006. Since 1992, he has been engaged in the research, standardization and development of wideband/broadband mobile radio communication technologies, terminals and systems, at NTT DOCOMO, INC., Kanagawa, Japan, and is currently engaged in the research of $5 \mathrm{G}$ radio access technologies and is promoting field trials of $5 \mathrm{G}$ system. He is a Leader of 5G Trial Promotion Group, the Fifth Generation Mobile Communications Promotion (5GMF). He is a senior member of The Institute of Electrical and Electronics Engineers, Incorporated (IEEE). 